\title{
Neurociencia del sueño: rol en los procesos de aprendizaje y calidad de vida
}

\author{
Neuroscience of sleep: \\ role in learning processes and quality of life
}

\author{
Luis A. Aguilar Mendoza 1*, Solange Caballero 2, Verónica Ormea 2, Ruth Aquino 2, Elena Yaya 2, Alan Portugal ', \\ Jose Gomez ' , Juana Zavaleta ', Ana Muñoz ${ }^{1}$ \\ 1 Universidad San Ignacio de Loyola, ${ }^{2}$ Universidad Peruana Cayetano Heredia
}

\section{RESUMEN}

El ritmo circadiano dura 24 horas en el cual están inmersas las horas de vigilia y sueño. El sueño es un estado fisiológico imprescindible para la calidad de vida en particular para el ser humano, igualmente para los procesos cognitivos como el aprendizaje; uno de los aspectos relevantes de esta consideración es la consolidación de la memoria de largo plazo. Presentamos, desde la perspectiva de la neurociencia, una revisión acerca del sueño y su relación con el aprendizaje y la calidad de vida. Luego se desarrolla la relación entre las estructuras sistémicas y las actividades neurofisiológicas, las cuales determinan o coadyuvan al logro de una buena calidad de vida. También nos referimos a los procesos de aprendizaje y su relación con el individuo (a nivel neuronal, cognitivo y conductual) como resultado de la interacción de éste con su entorno a través de las experiencias. Dentro de los procesos de aprendizaje, la memoria de corto plazo suele utilizarse en la vigilia mientras que y la consolidación se realiza en las fases profundas de sueño, mejorando en sí, la capacidad para adquirir, retener, almacenar y evocar información procesada. Uno de los aspectos a tener más en cuenta es que durante el sueño se reparan los circuitos cerebrales gracias a la neurogénesis post- embrionaria, que acompañada de una buena plasticidad adaptativa mejoran la calidad de vida de las personas a cualquier edad. La práctica de una buena "higiene de sueño", mejora los procesos de aprendizaje y la calidad de vida de las personas.

Palabras clave: Salud, aprendizaje, neurociencia, cerebro, sueño, memoria.

\begin{abstract}
The circadian rhythm lasts 24 hours in which wakefulness and sleep are immersed. The dream is a physiological state essential for the quality of life in particular for the human being, also for cognitive processes such as learning; One of the relevant aspects of this consideration is the consolidation of long-term memory. We present, from the perspective of neuroscience, a review of sleep and its relationship with learning and quality of life. Then we develop the relationship between systemic structures and neurophysiological activities, which determine or contribute to the achievement of a good quality of life. We also refer to the learning processes and their relationship with the individual (at the neuronal, cognitive and behavioral levels) as a result of their interaction with their environment through experiences. Within the learning processes, shortterm memory is usually used in wakefulness while and consolidation is carried out in the deep phases of sleep, improving this way, the ability to acquire, retain, store and evoke processed information. One of the aspects to take more into account is that during sleep, cerebral circuits are repaired thanks to post-embryonic neurogenesis, which, together with a good adaptive plasticity, improves the quality of life of people at any age. The practice of good "sleep hygiene" improves the learning processes and the quality of life of people.
\end{abstract}

Keywords: Health, learning, neuroscience, brain, sleep, memory.

Historial del artículo:

Recibido, 13 de marzo de 2017; aceptado, 1 de junio de 2017; disponible en línea, 05 de junio de 2017

* Doctor en neurociencias y biología del comportamiento, postdoctorado en neurociencia, y postdoctorado en neurofisiologia.

Correo: laguilar@usil.edu.pe 


\section{INTRODUCCIÓN}

A lo largo de la historia de la humanidad, el ser humano ha mostrado interés respecto al sueño. En la antigüedad se creía que mientras una persona dormía había una disminución o ausencia de la actividad cerebral (Cardinali, 2007). Actualmente, el sueño es considerado como un estado fisiológico, el cual es imprescindible para la vida humana y que si bien implica una disminución de la conciencia y una disminución al estado de alerta, durante el estado del sueño, se llevan a cabo procesos que requieren de la integración completa de la actividad cerebral y en el que se modifican muchos procesos fisiológicos del organismo (Bauzano, 2003). Mientras dormimos no respondemos a los estímulos visuales y nuestro umbral de respuesta a los estímulos auditivos aumenta (Aguilar et al. 2012).

Carrillo (2013) menciona algunas características conductuales asociadas al sueño, entre las que podemos citar: 1) es un proceso reversible, que difiere de otros estados como el estupor o el coma, 2) durante este periodo de descanso se produce una disminución de la conciencia y una reactividad a los estímulos externos, 3) el sueño se asocia a la inmovilidad y relajación muscular, 4) la ausencia de horas de sueño conlleva a alteraciones fisiológicas y conductuales, 5) durante este estado se asumen posturas estereotipadas y 6) responde al ritmo circadiano.

El sueño es un fenómeno periódico activo regular que se adapta a las variaciones biológicas del tiempo mediante ritmos biológicos como los infradianos, ultradianos y circadianos (Saavedra, Zúñiga, Navia y Vásquez, 2013).

El ritmo infradiano es aquel cuyas variaciones regulares son registradas en un tiempo mayor a 24 horas, mientras que en el ritmo ultradiano, son ciclos de menos de 24 horas (Tresguerres, Ariznavarreta, Cachofeiro, Cardinali, Escrich, Gil, Lahera, Mora, Romano y Tamargo, 2005). Los ritmos circadianos son ritmos biológicos u oscilaciones de las variables biológicas, con un periodo aproximado de 24 horas (Silva, 2010). Permiten reconocer los fenómenos de sueño- vigilia y su acción homeostática, en los procesos sistémicos de todo ser vivo (Saavedra et al, 2013).

El reloi biológico del ser humano rige los ritmos circadianos, así como el sueño y la vigilia. Se sitúa en el sistema nervioso central, en los núcleos supraquiasmáticos (NSQ) del hipotálamo. Las células del NQS se van a encargar de generar y coordinar los procesos fisiológicos rítmicos como el sueño - vigilia, la secreción de hormonas y la división celular (Hastings y Herzog, 2004).

Las neuronas del NSQ aumentan su actividad eléctrica durante el día, siendo la máxima hacia la tarde (aproximadamente hacia las 18:00 hrs.) precisamente cuando la melatonina comienza a liberarse reduciendo la actividad en el organismo. Es por ello, que se considera a la melatonina como la encargada de abrir las puertas al sueño (Cardinali, 2007).

El sueño tiene distintas fases a medida que pasa el tiempo al dormir, presentando diferentes procesos fisiológicos específicos en cada una de ellas, las cuales se complementan en ciclos repetitivos durante la noche. Entre estas etapas tenemos: NREM (Not rapid eyes movement) y REM (Rapid Eyes Movement), que se alternan sucesivamente entre cuatro a cinco veces por la noche. La fase de sueño NREM dura 6 horas y la fase de sueño REM 2 horas (Velayos, Moleres, Irujo, Yllanes y Paternain, 2007).

Esto se debe a que diferentes estructuras cerebrales controlan distintas fases del sueño. El tronco encefálico y el prosencéfalo basal están implicados principalmente en la regulación de las fases del sueño REM; mientras que, el área pre óptica ventrolateral del hipotálamo está implicada en la regulación del sueño NREM (Fernández - Mendoza y Puhl, 2014).

En cuanto a la actividad cerebral, en las primeras fases del sueño abundan ondas cerebrales lentas donde el movimiento de los ojos es lento y sin coordinación, por lo que a esta etapa también se le conoce como NREM; durante las últimas fases del sueño, por el contrario dormimos un sueño de ondas cerebrales rápidas, que se acompañan con movimientos y conjugados de ambos ojos, a esta fase se le denomina REM (Purves, Augustine, Fitzpatrick, Hall, LaMantia y White,2016).

Es importante señalar que tanto el sueño REM como NREM juegan un rol muy importante en la consolidación de memorias y el aprendizaje. Específicamente el sueño NREM o sueño de ondas lentas iniciaría este rol importante en el desarrollo y en el aprendizaje (Benington y Frank, 2003).

Velayo et al (2007) citando a McCarley (1995) y Pruves, Augustine, Fitzpatrick, Katz y La Mantia (2001), señala que para el estudio de los cambios funcionales que se dan durante el sueño se evalúan por medio del electroencefalograma (EEG), los movimientos oculares y el tono muscular. El registro de estos tres indicadores se denomina, polisomnografía. El sueño NREM está dividido en cuatro etapas o estadios.

La primera fase se caracteriza por la transición de la vigilia al sueño; y la segunda, porque la actividad cerebral se va haciendo más profunda. Aparecen patrones específicos de actividad cerebral llamados husos de sueño y complejos K. Tanto la temperatura, la frecuencia cardiaca y respiratoria comienzan a disminuir paulatinamente (Carrillo et al, 2013). 
Durante la tercera y cuarta fase, el sueño es más profundo, se ralentizan las ondas cerebrales. Esta fase es conocida como sueño lento.

El sueño REM, también llamado sueño paradóiico, se caracteriza por la disminución del tono muscular (con excepción de los músculos respiratorios y los esfínteres vesical y anal), así mismo la frecuencia cardiaca y respiratoria se vuelve irregular e incluso puede incrementarse; existe erección espontánea del pene o del clítoris (Carrillo et al, 2013).

Durante el sueño REM, el hipotálamo no ejerce la función de "termostato". Cuando se producen modificaciones extremas de la temperatura ambiente (calentamiento o enfriamiento pasivo), acontece un despertar o se produce el pase a una fase del sueño NREM (Aguilar et al, 2012).

\section{El sueño y el aprendizaje}

Hoy sabemos que es precisamente durante el sueño que se beneficia y facilita el mantenimiento neuronal, la neurogénesis (Guzman-Marín, Suntsova, Methippara, Greiffenstein, Szymusiak y McGint, 2005), así como el aprendizaje y la memoria (Huber, Ghilardi, Massini y Tononi, 2004), y la plasticidad cerebral. Es importante tener presente que el cerebro está genéticamente programado para desarrollarse potencialmente, pero para que pueda lograr su desarrollo pleno necesita de un ambiente que lo estimule adecuadamente $y$ positivamente.

Campos (2011) define el aprendizaje como un proceso que está relacionado con los cambios que ocurren en un individuo a nivel neuronal, cognitivo y conductual, como resultado de las experiencias permitiendo su adaptación al entorno.

Todos los seres humanos al nacer contamos con más conexiones y circuitos neuronales de los que necesitamos (exuberancia sináptica). Durante los dos primeros años se produce una poda programada en la cual el sistema nervioso central (SNC) se queda con aquellos circuitos que le son funcionales y útiles.

Esto no quiere decir que transcurrida esta etapa ya no se generen o modifiquen los circuitos, porque el sistema nervioso tiene la capacidad de responder a los estímulos extrínsecos e intrínsecos a través de la reorganización de su estructura, función y conexión, esto es conocido como la neuroplasticidad.

La plasticidad cerebral es un proceso de adaptación constante, mediante el cual las neuronas consiguen aumentar sus conexiones con las otras neuronas de forma estable a consecuencia de la experiencia, aprendizaje, estimulación sensorial y cognitiva (Aguilar, et al., 2012). Asimismo, debe ser entendida como la potencialidad del sistema nervioso de modificarse para formar conexiones nerviosas en respuesta a la disfunción o el daño (Garcés y Suárez, 2014).

Decimos que la plasticidad es expectante de la experiencia cuando el sistema nervioso está a la espera de estímulos que le permitan completar su desarrollo y funcionalidad. De ahí, la importancia de la experiencia (factores ambientales) para permitirle conformar, refinar y cablear determinados circuitos neuronales. Ejemplo: el movimiento comienza de lo más elemental y se van complejizando a lo largo de los años.

La plasticidad cerebral es dependiente de la experiencia porque está vinculada a mecanismos neurales que subyacen a la capacidad de un individuo para aprender a partir de sus experiencias personales, lo que le permite adaptarse a nuevos contextos personales, sociales y culturales.

Aguilar et al (2012) citando a Maquet, Smith y Stickgold (2003) señala que el sueño participa en funciones relacionadas con la plasticidad cerebral. Asimismo, citando a Hennevin, Huesz y Edeline (2007) menciona que esto guarda relación con la habilidad del cerebro para cambiar su estructura en respuesta al ambiente (reorganización y activación de las memorias).

Al hablar del aprendizaje no podemos dejar de lado a la memoria, ya que es esencial porque une los pensamientos y experiencias, permitiendo dar sentido y significado al comportamiento presente. Hace referencia a la capacidad para adquirir, retener, almacenar y evocar información del ambiente.

Gleichgerrcht (2013) señala tres procesos básicos que involucra la memoria:

- Codificar: Es cuando se incorpora la información y se registra en el cerebro.

- Almacenar: Es el proceso por el cual la información es guardada para luego ser encontrada.

- Evocación: Es la recuperación de la información cada vez que sea necesaria.

Todos los seres humanos tenemos la necesidad de guardar información en nuestros cerebros, pero como veremos más adelante no se lleva a cabo de la misma manera. Para poder ahondar en este tema, es necesario establecer la diferencia entre Sistemas de memoria y formas de memoria.

Las formas de memoria son parte de los sistemas de memoria, pueden ser: olfativas, visuales, verbales, etc. Mientras que los sistemas de memoria se encargan de procesar un tipo de información y conocimiento particular, se desarrollan en diferentes momentos de la vida y utilizan diferentes estructuras del cerebro. 
Para clasificar los sistemas de memoria, nos guiaremos de las propuestas de Squire (1992), Torralva y Sierra (2013) y Gleichgerrcht y Podestá (2013).

De acuerdo a los procesos básicos que involucra la memoria podemos dividirla en: memoria explícita (declarativa) y memoria implícita (no declarativa), cabe señalar que cada una se subdivide a su vez.

La memoria explícita es llamada también memoria declarativa. Entre las estructuras cerebrales que intervienen tenemos a la corteza prefrontal, amígdala, corteza perineal, diencéfalo, siendo el hipocampo una de las más resaltantes por ser la encargada de indicar cómo y dónde son guardadas las memorias, y puede recuperarlas cuando son necesarias. Esta memoria entra en funcionamiento cuando necesitamos recordar una experiencia pasada de manera consciente. Está conformada por la memoria episódica y la memoria semántica.

La memoria episódica se refiere a lugares y descripciones de eventos y personas. Están enmarcadas en un contexto real. Por ejemplo: cuando contamos una anécdota (tiene un comienzo, desarrollo y final). Dentro de esta memoria tenemos: la memoria de largo plazo (MLP) donde se guardan los aprendizajes significativos. Puede ser recuperado después de mucho tiempo de su aprendizaje. Y la memoria de trabajo (memoria online) este tipo de memoria trabaja con la información en cortos intervalos de tiempo. Por ejemplo: cuando hacemos cálculos mentales o recordamos un número telefónico antes de marcarlo, luego esta información se pierde.

A la memoria semántica debemos de entenderla como una gran enciclopedia o un diccionario de los significados de las palabras y las relaciones de estos significados. Están relacionados con experiencias concretas: hechos, números, símbolos, reglas gramaticales, fórmulas o reglas matemáticas.

La memoria implícita (no declarativa) es incidental o inconsciente. Está relacionada con aprendizajes condicionados y habilidades motoras. Incluye respuestas emocionales, habilidades, y hábitos, y respuestas a estímulos. Entra las estructuras cerebrales relacionadas tenemos a los ganglios basales, corteza premotora, y el cerebelo. Dentro de esta memoria podemos encontrar al priming y la memoria procedimental o procedural, pero en esta oportunidad nos centraremos solamente en la última.

La memoria procedimental o procedural tiene que ver con aprendizaje motor. Con la repetición de un acto motor éste mejora y se automatiza. Por ejemplo: manejar bicicleta: una persona que ha aprendido lo hace de manera automática (no piensa en cómo debe de pedalear). Lo mismo ocurre con la escritura, cuando hemos aprendido no pensamos en cómo se hace una grafía.

Esto lleva a formular la siguiente pregunta: ¿̇Cuál es la relación entre el sueño, el aprendizaje y la memoria? La relación entre el sueño, el aprendizaje y la memoria es muy compleja. Esto se puede deber en gran medida a que cada una de las fases del sueño parece relacionarse con un tipo de memoria.

A partir de las investigaciones celulares, moleculares, fisiológicos y conductuales que se han realizado tanto en animales como en seres humanos se ha propuesto que el sueño además de favorecer la consolidación de la memoria, facilita la adquisición de nueva información (Fernández et al., 2014).

Fernández et al. (2014) define la consolidación de la memoria como el proceso de estabilización de la experiencia sensoriomotora que ocurre tras la codificación de dicha información. Este proceso parece depender del sueño REM y de la Fase 2 del sueño NREM. La consolidación permite establecer conexiones entre los lóbulos temporales mediales y las áreas neocorticales del cerebro (McGaugh, 2000).

La evidencia científica indica que el sueño después del aprendizaje es fundamental para la consolidación de la memoria humana (Huber et al., 2004). Asimismo, se cree que el sueño antes del aprendizaje es igualmente esencial para la formación de nuevos recuerdos (Del Castillo y Mendoza, 2005). Una noche de privación de sueño produce un déficit significativo en la actividad del hipocampo durante la codificación de la memoria episódica, resultando en peor retención posterior. Durante el sueño se mejoraría la habilidad para recordar el lenguaje hablado, las habilidades motoras y la información de hechos (Aguilar et al., 2012).

La creciente evidencia sugiere que el sueño juega un papel importante en el proceso de aprendizaje de procedimientos. Más recientemente, el sueño se ha implicado en el desarrollo continuo de la habilidad motora después de la adquisición inicial (Walker, Brakefield, Seidman, Morgan, Hobson, y Stickgold, 2003).

Actualmente se cuenta con evidencias científicas que señalan que durante el sueño REM se favorece la memoria procedimental, ya que ésta puede ser mejorada. Asimismo, se ha podido corroborar que tiene un efecto beneficioso en la consolidación de las habilidades cognitivas como en las habilidades sensitivo-perceptuales. Mientras que el sueño NREM beneficia la memoria declarativa (hechos / episodios) (Born, Rasch, Gais, 2006).

En un estudio realizado por King et al. (2017) en personas mayores que presentaban un déficit en la 
consolidación de la memoria motora, se empleó fMRI para investigar los sustratos neuronales subyacentes a la consolidación de la memoria de la secuencia motora y la influencia moduladora de postaprendizaje del sueño, en adultos mayores sanos. Los participantes fueron entrenados en una secuencia motora $y$ reexaminados después de un intervalo de 8 horas incluyendo sueño de vigilia o diurno, así como un intervalo de 22 horas incluyendo una noche de sueño. Al concluir la investigación, los hallazgos no solo demostraron que el sueño posterior al aprendizaje puede mejorar la consolidación de la memoria motora en adultos mayores, sino que también proporcionan los correlatos neuronales a nivel de sistema de este efecto beneficioso.

Respecto a los neonatos y niños, Malasán, Sequieda y Ortiz (2013) indican que el sueño juega un papel fundamental para el desarrollo infantil, puesto que el requerimiento de horas de sueño depende de la etapa del ciclo vital y de las características de los niños. Por ejemplo, los recién nacidos duermen un promedio de 16 a 18 horas al día y hacia los 12 meses, la media de sueño es de unas 12-13 h al día.

Aguilar et al. (2012) citando a Tarullo, Balsam y Fifer (2011) señalan que el sueño óptimo prepara al infante para aprender cuando despierta, y después de que el aprendizaje ha ocurrido durante la vigilia, ya que los procesos centrales de memoria siguen durante el sueño, permitiéndole aprender respuestas adaptables a retos fisiológicos en el ambiente en el que duerme. Esto tiene implicancias para la supervivencia del infante.

Un estudio realizado en adolescentes muestra una conexión entre la pérdida de sueño y el rendimiento en la memoria de trabajo (Aguilar et al., 2012). Esto se debe a que los adolescentes tienen una cantidad insuficiente de sueño que se traduce en su habilidad para codificar, almacenar y recuperar información (Gradiar, Terrilll, Jhonston, y Douglas, 2008).

En la investigación llevada a cabo por McCanan, Bayliss, Pestell, Hill y Bucks (2016) respecto a la relación entre sueño y memoria de trabajo en niños con condiciones neurológicas, los resultados sugieren que la mala calidad del sueño se asocia con un componente ejecutivo de la memoria de trabajo verbal (más que espacial) en niños con condiciones neurológicas.

Lombardo et al. (2011) Ilevaron a cabo un estudio descriptivo - transversal en un grupo de estudiantes de preparatoria del sexo femenino de 15 a 18 años respecto a la relación entre los trastornos de sueño con rendimiento escolar, así como con el índice de masa corporal. Ellos concluyeron que la hipersomnia diurna afecta el rendimiento académico en un $0,7 \%$.
Los resultados en las materias de humanidades son satisfactorios gracias a la siesta que duermen las alumnas en el transporte escolar, ya que se relacionan con la memoria declarativa, mientras que las de ciencias no alcanzan a ser consolidadas por el tiempo que se requiere para entrar a sueño REM.

\section{Relación del sueño con las actividades neurofisiológicas}

El sueño es fundamental para la vida de los seres vivos y por ende, guarda una estrecha relación con las actividades neurofisiológicas. Para poder entender esto es importante tener en cuenta dos aspectos: a) en el dormir todas las fases del sueño son esenciales porque tienen una repercusión en el sistema nervioso a nivel fisiológico y morfológico; b) la deprivación del sueño llega a afectar la salud física y repercute en el entorno social, en el estado emocional y en la productividad de los individuos, entre otros.

\section{a) El sueño y el sistema inmunológico}

La dificultad para dormir debilita el sistema inmune, forzando los órganos, y exponiendo al individuo a un mayor riesgo de contraer enfermedades; además, incide en la fatiga del trabajo diario, disminuye la concentración y es causa de cefalea, artralgias y depresión (Lombardo, Velázquez, Flores, Casillas, Galván, García, Rosique y Rodríguez, 2011).

Se comprobó que la deprivación total de sueño por un periodo de 20 días producía la muerte en ratas. Dentro de los resultados, las ratas iban perdiendo y cambiando el color de su pelaje, se presentaron lesiones en la piel de la cola y las patas, así como una afectación en la alimentación (Cardinali, 2007).

En la actualidad se han incrementado los estudios respecto al sueño y su relación con el sistema inmunológico. Algunos de ellos han encontrado una relación entre el sueño y la respuesta de los anticuerpos a la hepatitis A cuando el sueño se encuentra alterado (Lange, Perras, Fehm, y Born, 2003).

Una de las investigaciones realizadas en seres humanos evidenció que dormir durante la noche después de haber recibido vacunas experimentales contra la hepatitis $A$ produce un fuerte y persistente aumento en el número de antígenos específicos de células Th, así como de anticuerpos. Juntos, estos hallazgos respaldan la idea de que el sueño de onda lenta contribuye a la formación de recuerdos biológicos a largo plazo y, en consecuencia, a respuestas adaptativas, conductuales e inmunológicas (Besedovsky, Lange, y Born, 2011).

En la actualidad se ha encontrado evidencias que el sueño sirve para la recuperación del metabolismo y de la homeostasis del organismo, para descansar 
(Fernández-Mendoza et al, 2014). El estudio llevado a cabo por Vera, Sánchez y Buelase (2007) centró en la relación entre Síndrome de Apnea Obstructiva del Sueño (SAOS) y el sistema inmune. Si bien sus resultados no son concluyentes, han encontrado evidencias que la apnea al ser una alteración del sueño puede tener efectos en el sistema inmune $y$, por ende, afectar la salud de las personas.

\section{b) El sueño y el sistema cardiovascular}

Durante el sueño NREM la frecuencia cardiaca, respiratoria y la presión arterial caen a niveles por debajo de los presentados durante el día. A diferencia del sueño REM, etapa en la que la presión arterial y la frecuencia cardiaca fluctúan.

Al despertar, independiente del período (uno puede despertarse para luego conciliar nuevamente el sueño), tanto la frecuencia cardíaca como la presión arterial aumentan. De acuerdo a las investigaciones realizadas las personas que padecen SAOS suelen presentar frecuencias altas de enfermedad coronaria.

Asimismo, el SAOS puede causar otros eventos fisiopatológicos en el sistema cardiovascular como: reducción en la liberación de oxígeno al miocardio, incremento en la demanda de oxígeno, isquemia miocárdica nocturna, edema pulmonar nocturno, hipertrofia del ventrículo izquierdo, disfunción izquierda e insuficiencia cardiaca (Araya, 2014).

\section{c) El sueño y el sistema endocrino}

De acuerdo a la información con la que contamos en la actualidad, la falta de sueño trae consigo alteraciones a nivel metabólico y endocrino. Por ejemplo, tenemos una disminución de la tolerancia a la glucosa, el aumento del nivel de grelina y disminución de los niveles de leptina, elevación de los niveles de cortisol (Canet, 2016) y una disminución de la hormona estimulante de la tiroides (García, 2016). En un reciente estudio realizado por Agüero y Haro (2016) para determinar si es que existe una asociación entre la deprivación de sueño nocturno durante la semana y el fin de semana, los hábitos alimentarios y la actividad física con el estado nutricional en escolares chilenos, se concluyó que sí existe una asociación entre menos horas de sueño e incremento del riesgo de sobrepeso/ obesidad. Cabe mencionar que al tratarse de un estudio transversal no se puede hablar de causalidad por lo que se recomienda un estudio longitudinal y de intervención para determinar si es que existe un efecto de mejora del estado nutricional cuando hay una adecuada higiene de sueño. La hormona del crecimiento se secreta en la primera hora de sueño, en la fase NREM. Además, los niveles más altos de secreción de la prolactina se observan durante el sueño REM, y la hormona tiroidea se secreta al final del día. Respecto a la secreción de las hormonas ACTH, del cortisol y de la adrenalina se producen al final del sueño, preparando al organismo para la vigilia (Aguilar et al, 2012). Con respecto a la hormona del crecimiento, el aumento de ésta durante el sueño lento apoyaría la hipótesis de que el sueño también cumpliría con una función restauradora. Aunque cabe señalar que en especies como los monos rhesus y los perros no se ha podido corroborar esta correlación (Tresguerres, et al, 2005).

\section{CONCLUSIONES}

La práctica diaria de una buena higiene de sueño, mejora los procesos de aprendizaje.

La buena calidad de sueño en las personas, mejora calidad de vida.

En la actualidad, las investigaciones que se están realizando en el campo de neurociencia nos permiten tener una mejor comprensión de todas las implicancias que tiene el sueño para la vida en especial para la del ser humano y abren nuevos senderos para futuros estudios.

En una época en la que se prioriza las horas de vigilia y se ha dejado de lado el tiempo destinado para el descanso, es necesario detenernos a reflexionar y crear conciencia sobre las consecuencias de la deprivación del sueño y la importancia de la higiene del sueño.

\section{REFERENCIAS BIBLIOGRÁFICAS}

Agüero, S. \& Haro, P. (2016). Asociación entre cantidad de sueño y obesidad en escolares chilenos. Arch Argent Pediatr, 114(2), 114-119.

Aguilar, L. Espinoza, G. Oruro, E. \& León, R. (2012). Breves consideraciones sobre el papel del sueño en la memoria y el aprendizaje. Módulo VI Curso 1 Factores que afectan el aprendizaje. Diplomado Neuropedagogía a distancia, CEREBRUM y ASEDH.

Araya, V. (2014). Apnea del sueño y enfermedad cardiovascular. Revista Costarricense de Cardiología, 16(1), 03-03.

Bauzano - Poley, E. (2003). El insomnio en la infancia. Revista de Neurología, 36, 381 - 390.

Benington, J. \& Frank, M. (2003). Cellular and molecular connections between sleep and synaptic plasticity. Prog. Neurobiol, 69(2). 71-101.

Besedovsky, L., Lange, T. y Born, J. (2012). Sleep and immune function. PflugersArchiv, 463(1), 121-137.

Born, J. Rasch, B., Gais, S. (2006). Sleep to remember. Neuroscientist, 12, 410-424.

Campos, A. L. (2011). Aprendizaje y Memoria. 
Separatas del Diplomado de Neuropedagogía. Lima: CEREBRUM- ASDEH.

Canet, T. (2016). Sueño y alimentación. Revista de Neurología, 63 (Suple 2), S17-S18.

Cardinali, D. (2007). Neurociencia Aplicada sus fundamentos. Buenos Aires: Editorial Médica Panamericana.

Carrillo, P., Ramírez, J. \& Magaña, K. (2013). Neurobiología del sueño y su importancia: antología para el estudiante universitario. Neurobiología del sueño, 56(4), 5-15.

Del Castillo, L. \& Mendoza, D. (2005). Algunos aspectos fisiológicos acerca del sueño. Revista de la Facultad de Ciencias de Salud. DUAZARI, 2(1), 57-64.

Fernández-Mendoza, J. \&Puhl, M. (2014). Capítulo 23 Sueño y aoursa. Neurociencia Cognitiva. Madrid: Médica Panamericana.

García - Borriquero, D. (2016). Principales retos en la investigación sobre hábitos del sueño. Revista de Neurología, 63(Suple 1), S21-S27.

Garcés, M. \& Suárez, J. (2014). Neuroplasticidad: aspectos bioquímicos y neurofisiológicos. Revista CES Medicina, 28(1), 119-131.

Gleichgerrcht, E. (2013). Memoria. Material entregado en la Maestría de Neurociencia y Educación de la Universidad Antonio Ruiz de Montoya y CEREBRUM.

Gleichgerrcht, E. \&M.G. de Podestá, M.E. (2013). Capítulo IV ¿̇Cómo aprendemos? El cerebro que aprende: Una mirada a la Educación desde la Neurociencia. Argentina: Aique.

Gradiar, M., Terrill, G., Jhonston, A. \& Douglas, P. (2008). Adolescent sleep and working memory performance. Sleep and Biological Rhythms, 6(3), 146-154.

Guzman-Marín, R., Suntsova, N., Methippara, M.,Greiffenstein, R., Szymusiak, R. \& McGint, D. (2005). Sleep depriv ationsuppresses neurogenesis in the adult hippocampus of rats. European Journal of Neuroscience, 22(8), $2111-2116$.

Hastings, M. \& Herzog, E. (2004). Clock Genes, Oscillators, and Cellular Networks in the Suprachiasmatic Nuclei. Journal of Biological Rhythms, 19(5), 400-413.

Huber, R., Ghilardi, M.F., Massini, M \&Tononi, G. (2004). Local sleep and learning. Nature, 430, 78 81.

King, B., Saucier, P., Albouy, G., Fogel, S., Rumpf, J., Klann, J., Buccino, G., Binkofski, F., Classen, J., Karni, A. \& Doyon, J. (2017). Cerebral activation during initial motor learning forecasts subsequent sleep-facilitated memory consolidation in older adults. Cerebral Cortex, 27,1588-1601.
Lange, T., Perras, B., Fehm, H. \& Born, J. (2003). Sleep enhances the human antibody response to hepatitis a vaccination. PsychosMed, 65, 831-835.

Lombardo, E.,Velázquez, J., Flores, G.,Casillas, G., Galván,A., García, P., Rosique, L. \& Rodríguez, L. (2011). Relación entre trastornos del sueño, rendimiento académico y obesidad en estudiantes de preparatoria. Acta Pediátrica de México, 32(3), 163-168.

Malasán, P., Sequeida, J. \& Ortiz, M. (2013). Sueño en escolares y adolescentes, su importancia y promoción a través de programas educativos. Revista Chilena de Pediatría, 84(5), 554-564.

McCanan,M., Bayliss, D., Pestell, C., Hill, C. \& Bucks, R. (2016). The relationship between sleep and working memory in children with neurological conditions. Child Neuropsychology. A journal on normal and abnormal development in childhood and adolescence. 1-18.

McGaugh, J. (2000). Memory a century of consolitain. Science, 287(5451), 248-251.

Purves, D., Augustine, Fitzpatrick, D., Hall, LaMantia, A.\& White (2016). Neurociencia ( $5^{\circ}$ ed.). Madrid: Médica Panamericana.

Saavedra, J., Zúñiga, L., Navia, C. y Vásquez, J. (2013). Ritmo circadiano: el reloj maestro. Alteraciones que comprometen el estado de sueño y vigilia en el área de la salud. Morfolia, 5(3), 16-35.

Silva, F. (2010). Trastornos del Ritmo Circadiano del Sueño: fisiopatología, clasificación y tratamientos. Revista Memoriza, 7, 1-13.

Squire,L. (1992). Declarative and Nondeclarative Memory: Multiple Brain Systems Supporting Learning and Memory. Journal of CognitiveNeuroscience, 4(3), 232-243.

Tresguerres, J., Ariznavarreta, C., Cachofeiro, V., Cardinali, D., Escrich, E., Gil, P., Lahera, V., Mora, F., Romano,

M. \& Tamargo, J. (2005). Fisiología Humana ( $3^{\circ}$ ed.). Madrid: McGraw Hill.

Velayos, J., Moleres, F., Irujo, A., Yllanes, D. \& Paternain, B. (2007). Bases anatómicas del sueño. Anales del sistema sanitario de Navarra, 30 (Suplemento 1),7-17.

Vera, P., Sánchez, A.\&Buela, G. (2007). Sueño y Sistema Inmune: Diferencias en Variables Inmunológicas en Sujetos con Apnea y Controles. Revista Ecuatoriana de Neurología, 16(3), 181-184.

Walker, M., Brakefield, T., Seidman, J., Morgan, A., Hobson, J. \& Stickgold, R. (2003).Sleep and the time course of motor skill learning. Learning Memory, 10(4), 275-284. 